\title{
Metastatic Peripheral Neuroblastoma in a Dog
}

\author{
Fernando Fernandes Sayeg, Mariana Correia Oliveira, Bartolomeu Benedito Neves dos Santos, \\ Mariana Siqueira d'Ávila, Thiago Souza Costa, Júlio Israel Fernandes, Max Ferreira de Andrade \\ \& Vivian de Assunção Nogueira
}

\begin{abstract}
Background: Peripheral neuroblast neoplasms are considered as a group of tumors derived from primitive cells of the neural crest that are progenitors of the sympathetic ganglia and adrenal medulla. Reports of neuroblastoma in dogs are scarce in English literature, and there are no reports from Brazil due to its rare occurrence or missed diagnosis, because modern techniques are often not accessible in Brazil. The aim of the present study was reported a case of metastatic peripheral neuroblastoma in a 10-month-old bitch of Canadian Shepherd breed.

Case: A 10-month-old female dog, Canadian Shepherd breed, presenting prostration, inappetence, emesis, progressive weight loss, and difficulties in the locomotion of the pelvic limbs was brought to the Small Animal Veterinary Hospital of the Federal Rural University of Rio de Janeiro (HVPA / UFRRJ) for treatment. Palpation of the abdominal cavity revealed a mass of approximately 14.0-cm diameter in the thoracolumbar region. After the second visit to HVPA/ UFRRJ, there was worsening of the clinical condition and onset of pain and dyspnea; hence, euthanasia was performed. The dog was referred for necropsy; the examination revealed a mass measuring $40.0 \times 35.0 \mathrm{~cm}$ in the abdominal cavity that caused displacement of the intestines and compressed the liver against the diaphragm, as well as another mass that traversed the diaphragm through the esophageal hiatus and surrounded the esophagus, aorta, and caudal vena cava. The right adrenal gland was compressed and adhered to the mass, and the left adrenal gland was not visualized. Specimens of various organs were collected, fixed in $10 \%$ buffered formalin, and processed according to routine histological technique. Immunohistochemical examination performed on mass specimens revealed positivity for the anti-CD56, anti-synaptophysin, anti-GFAP, and anti-NSE markers and negativity for the anti-chromogranin and anti-Olig2 markers. Based on pathology and immunohistochemistry findings, a diagnosis of poorly differentiated peripheral neuroblastoma was made; in addition, presence of emboli of neoplastic cells in the hepatic sinusoids and branches of the portal vein and metastasis in the spleen, lymph nodes, lung, and meninges were confirmed.

Discussion: Positive results for neural markers associated with negative results for those of other small round cell tumors was considered to indicate the presence of neuroblast tumors; glioblastoma and oligodendroglioma were ruled out based on the absence of anti-Olig2 labeling, and, especially, the possibility of occurrence of pheochromocytoma was excluded based on the absence of anti-chromogranin. During necropsy, the location of the mass at the retroperitoneal space near the thoracolumbar junction, craniomedially in relation to the left kidney, was confirmed, which is compatible with the location of the adrenal gland. Metastasis in the dura mater was determined to be present based on necropsy findings alone, and neoplastic invasion through the esophageal hiatus of the diaphragm of a large part of the thoracic cavity was observed, which, to the best of our knowledge, have not been described in reports of Veterinary Medicine. The high rate of tumor growth and compression and invasion of adjacent tissues and organs may be considered as potential factors for unfavorable prognosis of peripheral neuroblastoma.
\end{abstract}

Keywords: neuroblastoma, neural crest, canine. 


\section{INTRODUCTION}

Peripheral neuroblast neoplasms are considered as a group of tumors derived from primitive cells of the neural crest; such cells are responsible for forming the sympathetic ganglia and the medullary adrenal region. The frequency and pathogenesis of neuroblastoma in animals are unknown $[3,9,19]$; in contrast, in humans, it is considered a common neoplasia mainly in children up to 2 years old [12] and involves pathogenetic factors of exposure to environmental factors, prenatal alcohol consumption, use of pesticides, and phenobarbital [2].

Clinical signs are nonspecific and include lethargy, anorexia, pain, fever, and weight-loss. Other less common signs are derived from tumor development and consequent deleterious effects of compression of the adjacent organs [5].

Generally, peripheral neuroblastoma is characterized by formation of large abdominal masses with high capacity for infiltration and metastasis [6].

In human medicine, treatment is selected according to the International Neuroblastoma Pathological Classification (INSS) status [12,18]; whereas, in veterinary medicine, there are no reports of treatment options or attempts in patients with peripheral neuroblastoma, due to the fact that euthanasia is usually performed before confirming the diagnosis, or the guardian does not seek further clinical care $[1,3,6-10,13]$.

In this study, we reported our experience of a case of metastatic peripheral neuroblastoma in a 10-month-old bitch of Canadian Shepherd breed.

\section{CASE}

A 10-month-old bitch, Canadian Shepherd breed, was brought to the Small Animal Veterinary Hospital of the Federal Rural University of Rio de Janeiro (HVPA / UFRRJ) for treatment. The guardian reported that for 4 months prior to visit, the patient had presented prostration, inappetence, emesis, progressive weight loss, and difficulties in locomotion of the pelvic limbs.

Clinical examination revealed moderate cachexia and alert state of consciousness, and temperature of $38^{\circ} \mathrm{C}$, heart rate of $124 \mathrm{bpm}$, and respiratory rate of $36 \mathrm{rpm}$. The mucosae were normocorated and no enlarged superficial lymph node was observed; palpation of the abdominal cavity revealed a mass of soft consistency, approximately $14.0 \mathrm{~cm}$ in diameter, located in the thoracolumbar region and slightly displaced to the right side. Based on clinical findings, radiography of the spine and abdominal ultrasonography were performed; the results revealed decreases in the intervertebral spaces of the lumbar vertebrae, diffuse splenomegaly, and increased volume in the soft tissues located in the third of the right thoracic region, and initial third of the right dorsal lumbar region, without bone involvement.

Analgesic therapy with dipyrone ${ }^{1}(25 \mathrm{mg} / \mathrm{kg}$ tid), tramadol hydrochloride ${ }^{2}$ (4 mg/kg bid), carprofen ${ }^{3}$ ( $4.4 \mathrm{mg} / \mathrm{kg} \mathrm{sid})$, and supportive treatment with omeprazole $^{4}(1 \mathrm{mg} / \mathrm{kg} \mathrm{sid})$, and cobavital ${ }^{5}(0.4 \mathrm{mg} / \mathrm{kg})$ were administered. The hemogram revealed normocytic normochromic anemia and leukopenia due to lymphopenia, both discrete; serum biochemistry showed a slight decrease in alkaline phosphatase, alanine aminotransferase, and aspartate aminotransferase.

Fine needle aspiration cytology was performed, and findings obtained indicated low cellularity and inconclusive diagnosis. Subsequently, incisional biopsy of the patient under anesthesia was performed with a tru-cut and punch needle of $3.0 \mathrm{~mm}$ under ultrasonography guidance. Two fragments were collected and fixed in $10 \%$ buffered formalin and sent to the Pathology Department of the Federal Rural University of Rio de Janeiro (SAP / UFRRJ).

Histopathological examination revealed proliferation of neoplastic cells with indistinct cytoplasmic boundaries, sparse and discreetly eosinophilic cytoplasm, and an oval nucleus with condensed or coarsely granular chromatin, with the nucleolus not visualized; in addition, moderate anisocytosis and anisokaryosis and mitotic figures in 10 fields of greatest magnification (400x) were observed. Based on these findings, poorly differentiated sarcoma was diagnosed.

Due to worsening of the patient's clinical condition and onset of pain and dyspnea, euthanasia was performed by the tutors. The animal was referred for necropsy. The examination revealed a mass of 40.0 $\mathrm{x} 35.0 \mathrm{~cm}$ in the abdominal cavity, with a reddish-colored, multinodular irregular external surface that caused displacement of the small and large intestines and compression of the liver against the diaphragm; moreover, there was spread of the mass through the esophageal hiatus of the diaphragm, surrounding the esophagus, aorta, and caudal vena cava. The thoracic portion of the mass was $20.0 \times 10.0 \mathrm{~cm}$, with the same 
morphological characteristics as that in the abdominal cavity; the right adrenal gland was compressed and adhered to the tumor, whereas, the left adrenal was missing; mass infiltration at the dorsal muscles and vertebral canal, and compression of the spinal cord at level of T10 to L5 was observed. Weight of tumor on removal from the abdominal cavity was $2.0 \mathrm{~kg}$, and soft consistency on cutting with reddish tint of compact cut surface was noted. At the opening of the skull, three circumscribed areas of $0.5 \mathrm{~cm}$ in the dura were visualized, with irregular slightly rough surface and reddish tonality.

Specimens of the tumor and various tissues (the adrenal, liver, lymph nodes, heart, lung, stomach, intestine, pancreas, spleen, kidney, bladder, meninges, and central nervous system) were collected, fixed in $10 \%$ buffered formalin, and processed according to routine histological technique; paraffin blocks containing fragments of the neoplasia were submitted for immunohistochemistry including the following markers: anti-CD56, anti-synaptophysin, anti-GFAP, anti-NSE (enolase specific neuron), anti-chromogranin, and anti-Olig2.

Microscopy revealed proliferation of neoplastic cells, densely cellular lesion arranged in nests, and packages supported by fine fibrovascular stroma and separated into lobes by thick fibrous septa. Neoplastic cells were oval to polygonal, with moderately distinct limits, and moderate cytoplasm slightly eosinophilic and granular. The nucleus was round to oval, and characterized as hyperchromatic or with chromatin finely dotted with one to two visible nucleoli. Pleomorphism, anisocytosis, and moderate anisokaryosis were observed, and seven mitosis figures were visualized per magnification (400x) field. Areas of central necrosis were present in some neoplastic cell nests (Figures 1-2).

Immunohistochemical examination revealed positivity for anti-CD56 (Figure 3), anti-synaptophysin (Figure 4), anti-GFAP (Figure 5), and anti-NSE (Figure 6) markers, and negativity for anti-chromogranin (Figure 7) and anti-Olig2 (Figure 8) markers.

Based on the pathology and immunohistochemistry findings of a neoplasm with predominance of neuroblasts with $<5 \%$ of ganglion cells and low Schwannoma tumor, poorly differentiated peripheral neuroblastoma was diagnosed; presence of emboli of the cells in the hepatic sinusoids and branches of the
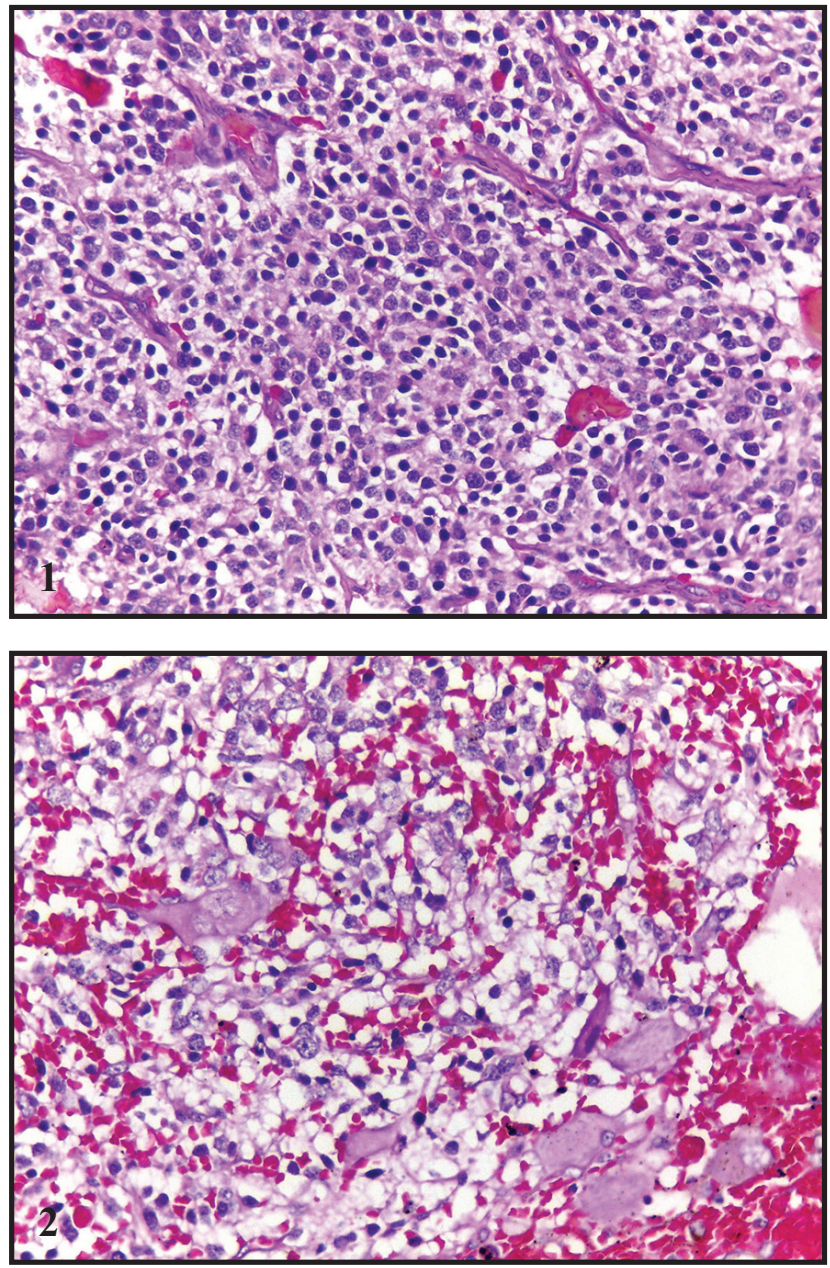

Figures 1 \& 2. Peripheral neuroblastoma in a Canadian Shepherd Dog. Clusters of tightly packed cells divided by moderated fibrovascular stroma. Neoplastic cells have moderate distinct borders and scant cytoplasm [HE; 400x].

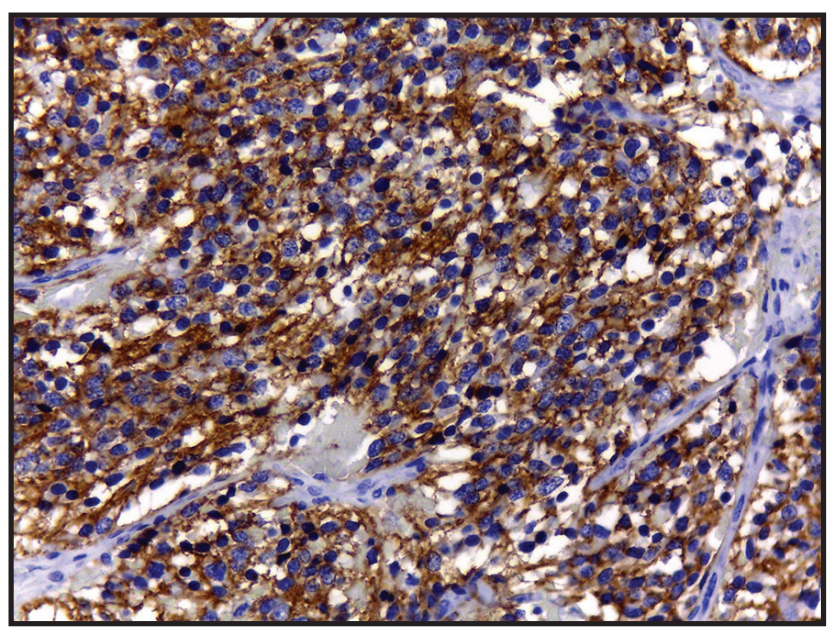

Figure 3. Diffuse and strong perimembranous and membranous labeling for CD56 in the neuroblastic cells [CD56; 40x].

portal vein, and metastasis in the spleen, lymph nodes, lung, and meninges were confirmed. 


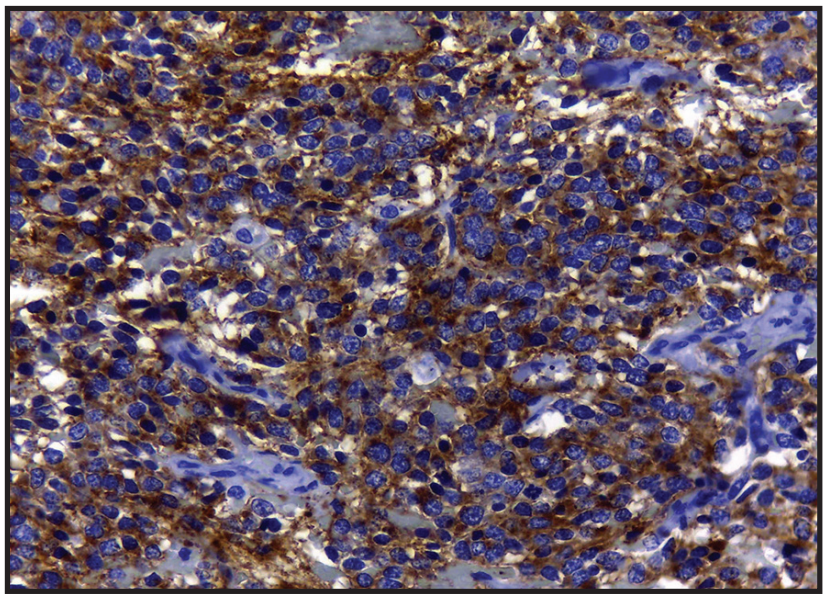

Figure 4. Diffuse and strong perimembranous and membranous labeling for and anti-synaptophysin [40x]

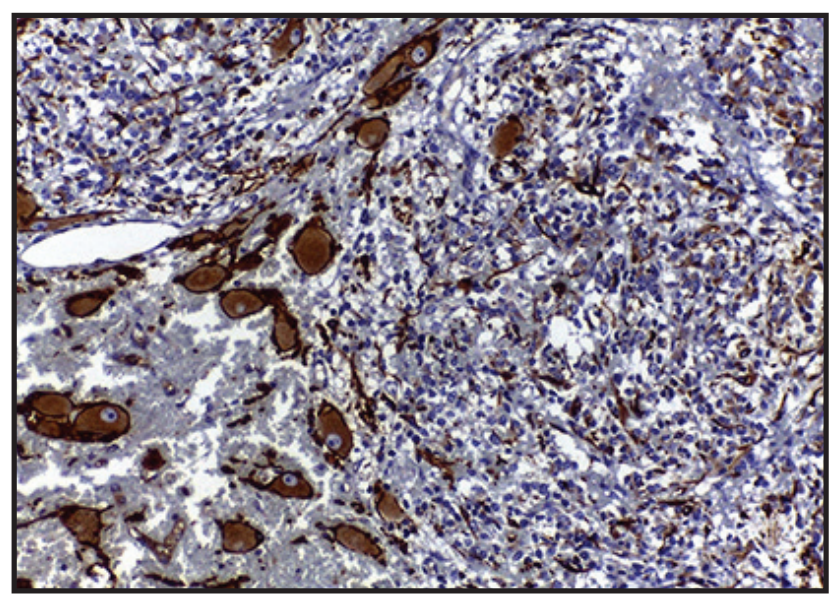

Figure 5. Mature neuronal cells showed strong membranous immunoreactivity for GFAP [20x].

\section{DISCUSSION}

In this case, the diagnosis of peripheral neuroblastoma was made based on the findings of pathology and immunohistochemistry. The tumor was positive for anti-CD56, anti-GFAP, anti-synaptophysin, and anti-NSE antibodies and negative for anti-chromogranin and anti-Olig2. Positive results for neural markers combined with negative results for other markers of small round cell tumors were considered to indicate the presence of neuroblast tumors [12], and the absence of anti-Olig2 labeling was considered to rule out glioblastoma and oligodendroglioma; especially, the possibility of occurrence of pheochromocytoma was excluded based on the absence of anti-chromogranin.

By histological definition, neuroblastoma is a tumor comprising neuroblasts arranged in groups with or without proliferation of Schwann cells. Differential diagnoses include peripheral neuroblast tumors, such

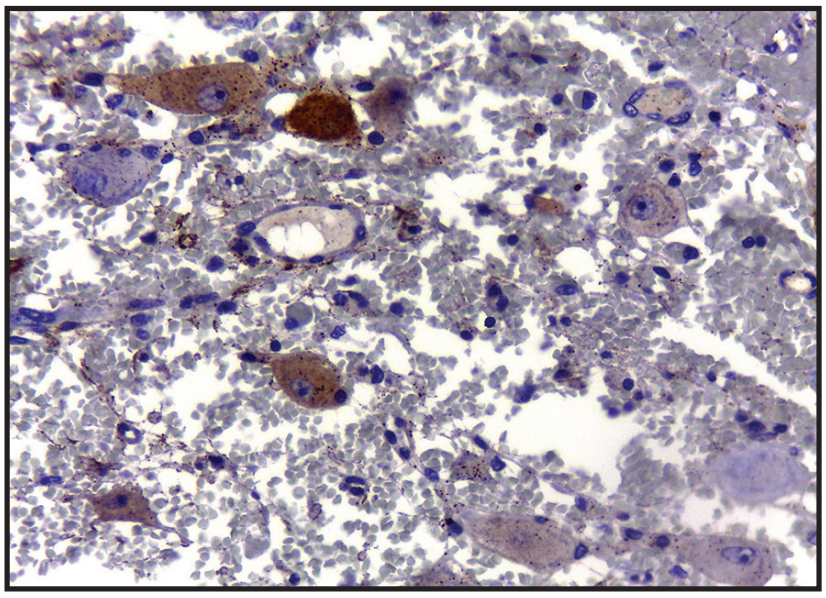

Figure 6. Neuronal cells showed immunoreactivity diffuse cytoplasmic labeling for NSE [40x].

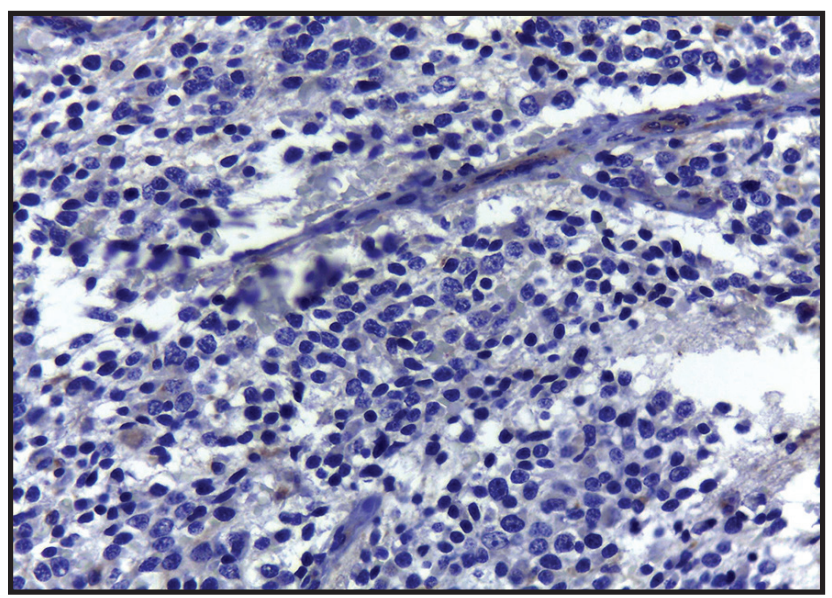

Figure 7. Neoplastic cells were negative for Olig2 [40x].

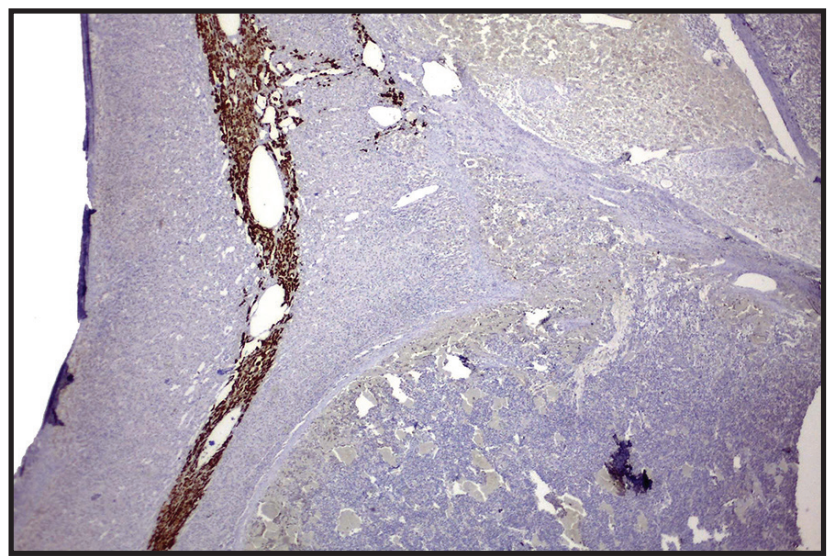

Figure 8. Chromaffin cells were positive for chromogranin A in the adrenal medulla. Neoplastic cells failed to express immunoreactivity [Chromogranin; 5x].

as ganglioneuroblastoma and ganglioneuroma [1]. Ganglioneuroblastoma is characterized by foci of neuroblasts at various stages of differentiation randomly mixed with mature ganglion cells and arranged on abundant Schwannian stroma [17]. 
Peripheral neuroblastoma in dogs remains unclear due to scarcity of reports in English literature, and absence of reported studies in Brazil which reflects rare incidence of this neoplasm.

In our case, the patient age was $<1$ year, similar to that of cases described previously with patient age $<2$ years [6-10,13]. Reports have indicated diagnosis of peripheral neuroblastoma in dogs aged 4,8 , and 10 years $[1,3]$.

Diarrhea may occur due to ability of this tumor to secrete vasoactive intestinal polypeptides, as in patients with Kerner-Morrison syndrome [16].

During necropsy, location of the mass at retroperitoneal space, near the thoracolumbar junction, craniomedially in relation to the left kidney was noted, which was compatible with location of the adrenal gland. The pluripotent cells are the tumors' source and migrate from the neural crest to form the sympathetic ganglia, paragranules, and medullary adrenal region [12].

In our patient, metastasis in the dura mater was detected based on necropsy findings alone, and invasion of the neoplasm through the esophageal hiatus of the diaphragm and its wide spread within the thoracic cavity was observed; to the best of our knowledge, this has not been previously described in the literature, and occurrence of epidural and intradural metastasis has only been reported in humans [4].
In microscopic analysis, a small number of individualized cells were observed, with abundant cytoplasm and eosinophilic and large and oval nuclei compatible with ganglion cells $[1,3,10]$.

No cases of peripheral neuroblastoma in veterinary medicine have been managed with treatment modalities used in human medicine, such as surgery, chemotherapy, and radiotherapy; in some cases, the patient died due to anesthesia performed during the biopsy, or it was not feasible to perform the treatment due to poor clinical conditions or high treatment cost. Considering these situations combined with the high rate of tumor growth, and compression and invasion of the adjacent tissues and organs, peripheral neuroblastoma is considered to have an unfavorable prognosis $[11,16]$.

\section{MANUFACTURERS}

${ }^{1}$ Laboratório Teuto Brasileiro S.A. Anápolis, GO, Brazil.

${ }^{2}$ Brainfarma Indústria Química e Farmacêutica S.A. Anápolis, GO, Brazil.

${ }^{3}$ Agener União Química Farmacêutica Nacional S.A. Pouso Alegre, MG, Brazil.

${ }^{4}$ Laboratório Globo Ltda. São José da Lapa, MG, Brazil.

${ }^{5}$ Abbott Laboratórios do Brasil Ltda. São Paulo, SP, Brazil.

Declaration of interest. Declaration of interest. The authors report no conflicts of interest. The authors alone are responsible for the content and writing of the paper.

\section{REFERENCES}

1 Arenas-Gamboa A.M., Tanabe M., Edwards J. \& Storts R. 2014. Peripheral neuroblastomas in dogs: a case series. Journal of Comparative Pathology. 150(4): 361-365.

2 Belson M., Kingsley B. \& Holmes A. 2007. Risk factors for acute leukemia in children: a review. Environmental Health Perspectives. 115(1): 138.

4 Cook R.W., Abraham L.A. \& McCowan C.I. 2017. Disseminated peripheral neuroblastoma in a Rhodesian Ridgeback dog. Australian Veterinary Journal. 95(4): 129-133.

5 De Bernardi B., Rogers D. \& Carli M., Madon E., Laurentis T., Bagnulo S., Di Tullio M.T.B., Paolucci G. \& Pastore G. 1987. Localized neuroblastoma: surgical and pathologic staging. Cancer. 60(5): 1066-1072.

6 Evans A.E. Chatten J. \& D'Angio G.J., Gerson, J.M., Robinson J. \& Schnaufer L. 1980. A review of 17 IV-S neuroblastoma patients at the Children's Hospital of Philadelphia. Cancer. 45(5): 833-839.

7 Forrest L.J. Galbreath E.J., Dubielzig R.R. \& MacEwen E.G. 1997. Peripheral neuroblastoma in a dog. Veterinary Radiology \& Ultrasound. 38(6): 457-460.

8 Kelly D.F. 1975. Neuroblastoma in the dog. The Journal of Pathology. 116(4): 209-212.

9 Louden C., Patterson J.S. \& Sandusky G.E. 1992. Peripheral neuroblastomas in two dogs. Journal of Veterinary Diagnostic Investigation. 4(4): 476-480.

10 Marcotte L., McConkey S.E., Hanna P., Foley P. \& Burton S. 2004. Malignant adrenal neuroblastoma in a young dog. The Canadian Veterinary Journal. 45(9): 773.

11 Matsushima S., Maruyama T. \& Tor M. 1998. Peripheral neuroblastoma in a young beagle dog. Toxicologic Pathology. 26(6): 806-809. 
12 Matthay K.K., Perez C., Seeger R.C., Brodeur G.M., Shimada H. \& Atkinson J.B. 1998. Successful treatment of stage III neuroblastoma based on prospective biologic staging: a Children's Cancer Group study. Journal of Clinical Oncology. 16(4): 1256-1264.

13 Park J.R., Eggert A. \& Caron H. 2008. Neuroblastoma: biology, prognosis, and treatment. Pediatric Clinics of North America. 55(1): 97-120.

14 Payne-Johnson C.E. \& Brockman D.J. 1992. Neuroblastoma in the dog. Journal of Small Animal Practice. 33(8): 395-398.

15 Perez C.A., Matthay K.K., Atkinson J.B., Seeger R.C., Shimada H., Haase G.M., Stram D.O., Gerbing R.B. \& Lukens J.N. 2000. Biologic variables in the outcome of stages I and II neuroblastoma treated with surgery as primary therapy: a children's cancer group study. Journal of Clinical Oncology. 18(1): 18.

16 Scheibel E., Rechnitzer C., Fahrenkrug J. \& Hertz H. 1982. Vasoactive intestinal polypeptide (VIP) in children with neural crest tumours. Acta Pediatrica. 71(5): 721-725.

17 Schmidt M.L., Lukens J.N., Seeger R.C., Brodeur G.M., Shimada H., Gerbing R.B., Stram D.O., Perez C., Haase G.M. \& Matthay K.K. 2000. Biologic factors determine prognosis in infants with stage IV neuroblastoma: a prospective Children's Cancer Group study. Journal of Clinical Oncology. 18(6): 1260-1268.

18 Shimada H., Chatten J., Newton Jr. W.A., Sachs N., Hamoudi A.B., Chiba T., Marsden H.B. \& Misugi K. 1984. Histopathologic prognostic factors in neuroblastic tumors: definition of subtypes of ganglioneuroblastoma and an agelinked classification of neuroblastomas. JNCI: Journal of the National Cancer Institute. 73(2): 405-416.

19 Shimada H., Ambros I.M., Dehner L.P., Hata J.I., Joshi V.V., Roald B., Stram D.O., Gerbing R.B., Lukens J.N., Matthay K.K. \& Castleberry R.P. 1999. The international neuroblastoma pathology classification (the Shimada system). Cancer. 86(2): 364-372.

20 Steinberg H., Peek S.F. \& Nelson K.M. 2006. Neuroblastoma with neuronal differentiation in the spinal cord in an Aberdeen Angus heifer calf. Veterinary Pathology. 43(2): 193-197. 